\title{
A DENSITY THEOREM WITH AN APPLICATION TO GAP POWER SERIES( $\left.{ }^{1}\right)$
}

\author{
BY \\ K. G. BINMORE
}

Abstract. Let $N$ be a set of positive integers and let

$$
F(z)=\sum A_{n} z^{n}
$$

be an entire function for which $A_{n}=0(n \notin N)$. It is reasonable to expect that, if $D$ denotes the density of the set $N$ in some sense, then $F(z)$ will behave somewhat similarly in every angle of opening greater than $2 \pi D$. For functions of finite order, the appropriate density seems to be the Pólya maximum density $\mathscr{P}$. In this paper we introduce a new density $\mathscr{D}$ which is perhaps the appropriate density for the consideration of functions of unrestricted growth. It is shown that, if $|I|>2 \pi \mathscr{D}$, then

$$
\log M(r) \sim \log M(r, I)
$$

outside a small exceptional set. Here $M(r)$ denotes the maximum modulus of $F(z)$ on the circle $|z|=r$ and $M(r, I)$ that of $F\left(r e^{i \theta}\right)$ for values of $\theta$ in the closed interval I. The method used is closely connected with the question of approximating to functions on an interval by means of linear combinations of the exponentials $e^{i x n}$ $(n \in N)$.

1. Introduction. Let $S$ be a normed vector space over the complex field. If $T \subset S$, let $V(T)$ denote the subspace of $S$ generated by $T$.

Let $E=\left\{e_{n} \mid n=0,1, \ldots\right\}$ be a linearly independent subset of $S$ and, if $x \in V(E)$, write $x=\sum x_{n} e_{n}$, where all but a finite number of terms are zero.

The set $E$ is said to be free in $S$ if no element of $E$ belongs to the closure of the vector subspace generated by the other elements of $E$. A necessary and sufficient condition that $E$ be free in $S$ is the existence of positive constants $C_{n}(n=0,1, \ldots)$ with the property that

$$
\left|x_{n}\right| \leqq C_{n}\|x\| \quad(n=0,1, \ldots)
$$

whenever $x \in V(E)$. It follows that, if $E$ is free in $S$, each $x \in \operatorname{cl} V(E)$ has a unique formal expansion $x \sim \sum x_{n} e_{n}$. Here $x_{n}=L_{n}(x)$, where each $L_{n}(n=0,1, \ldots)$ is a continuous linear functional on $\mathrm{cl} V(E)$.

The set $E$ is said to be fundamental in $S$ if $V(E)$ is dense in $S$.

Received by the editors August 21, 1968 and, in revised form, August 21, 1969.

AMS Subject Classifications. Primary 3020; Secondary 4115.

Key Words and Phrases. Gap power series, entire functions, closure, completeness and freedom, $\mathscr{L}^{p}$ spaces, Beurling-Malliavin density, Pólya maximum density, Fourier transforms.

( ${ }^{1}$ Supported by National Science Foundation Grant No. GP-8199.

Copyright (C) 1970, American Mathematical Society 
In this paper, we particularise $S$ to be one of the spaces $\mathscr{L}^{p}(-\pi \Delta, \pi \Delta)$, where $1 \leqq p \leqq \infty$ and $0<\Delta \leqq 1$. We let $\Lambda=\left\{\lambda_{n}\right\}$ be a strictly increasing sequence of positive integers and take the elements of $E=E_{\Lambda}$ to be

$$
e_{n}=\exp \left(i x \lambda_{n}\right) \quad(n=0,1, \ldots)
$$

It was shown by $L$. Schwartz [14] that, if $E_{\Lambda}$ is nonfundamental in $\mathscr{L}^{p}(-\pi \Delta, \pi \Delta)$, then $E_{\Lambda}$ is free in $\mathscr{L}^{p}(-\pi \Delta, \pi \Delta)$. Further, if $E_{\Lambda}$ is free in $\mathscr{L}^{p}(-\pi \Delta, \pi \Delta)$ and $\Delta^{\prime}>\Delta$, then $E_{\Lambda}$ is nonfundamental in $\mathscr{L}^{p}\left(-\pi \Delta^{\prime}, \pi \Delta^{\prime}\right)$.

As before we have that, if $E_{\Lambda}$ is free in $\mathscr{L}^{p}(-\pi \Delta, \pi \Delta)$, then there exist positive constants $C_{n}(n=0,1, \ldots)$ such that $\left({ }^{2}\right)$

$$
\left|a_{n}\right| \leqq C_{n}\|f\|_{p} \quad(n=0,1, \ldots),
$$

whenever $f$ is a function of $\mathrm{cl} V(E)$ with expansion

$$
f \sim \sum_{n=0}^{\infty} a_{n} e^{i x \lambda_{n}} .
$$

In attempting to use inequality (1.1), two questions arise.

(1) What structural properties of the sequence $\Lambda$ imply that $E_{\Lambda}$ is free in $\mathscr{L}^{p}(-\pi \Delta, \pi \Delta)$ ?

(2) Given that $E_{\Lambda}$ is free in $\mathscr{L}^{p}(-\pi \Delta, \pi \Delta)$, how large are the constants $C_{n}$ $(n=0,1, \ldots)$ ?

An answer to (1) has been given by Beurling and Malliavin [2]. For an expository account of their work, see Kahane [8]. For each sequence $\Lambda$ they construct a number $\mathscr{B}$ (which we call the Beurling-Malliavin density of $\Lambda$ ) with the property that $E_{\Lambda}$ is fundamental in $\mathscr{L}^{p}(-\pi \Delta, \pi \Delta)$ if $\Delta<\mathscr{B}$ and nonfundamental (and therefore free) if $\Delta>\mathscr{B}$.

Some estimates for the constants $C_{n}$ in (2) have been obtained in [1], [3] and [4] under various hypotheses. (See also [5] and [7].) In this paper we obtain another theorem of this type and discuss an application it has to the theory of entire functions with gap power series. The proof of the theorem is elementary and does not depend on the work of Beurling and Malliavin.

2. Density conditions. The Pólya maximum density $\mathscr{P}$ (see e.g. Levinson [11]) of a sequence $\Lambda$ may be defined in the following way. Let $\Gamma$ denote the class of functions $\gamma(x)=\eta x(0<\eta<1)$. Then

$$
\mathscr{P}=\sup _{\gamma \in \Gamma} \limsup _{x \rightarrow \infty} \frac{N[x-\gamma(x), x)}{\gamma(x)}
$$

where $N[a, b)=N(I)$ denotes the number of terms of $\Lambda$ contained in the interval $I=[a, b)$. We have that $\mathscr{P} \leqq \mathscr{B}$.

$\left.{ }^{2}\right)$ Except where otherwise noted $\|f\|_{p}=\left\{\int_{-\pi \Delta}^{\pi \Delta}|f(x)|^{p} d x\right\}^{1 / p}$, with the usual understanding for the case $p=\infty$. 
We now introduce a new density condition which is closely related to the Beurling-Malliavin density $\mathscr{B}$ but defined in a similar way to $\mathscr{P}$.

Let $\Psi$ denote the class of functions $\psi$ which are positive and continuous on $(1, \infty)$ and which satisfy

(1) $\quad \psi(x) \uparrow \infty$;

(2) $\quad x^{-1} \psi(x) \downarrow 0$;

(3) $\quad \int_{1}^{\infty} x^{-2} \psi(x) d x<\infty$.

We then define the " $\Psi$-density" $\mathscr{D}$ of $\Lambda$ by

$$
\mathscr{D}=\inf _{\psi \in \Psi} \limsup _{x \rightarrow \infty} \frac{N[x-\psi(x), x)}{\psi(x)} .
$$

We have $\mathscr{P} \leqq \mathscr{B} \leqq \mathscr{D}$.

3. Statement of results.

THEOREM 1. Suppose $\Lambda$ has $\Psi$-density $\mathscr{D}$ and $\mathscr{D}<\Delta<1$. Then $E_{\Lambda}$ is free in $\mathscr{L}^{p}(-\pi \Delta, \pi \Delta)(1 \leqq p \leqq \infty)$, and there is $a \psi \in \Psi$, which depends only on $\Lambda$ and $\Delta$, such that

$$
\left|a_{n}\right| \leqq \exp \left(\psi\left(\lambda_{n}\right)\right)\|f\|_{p} \quad(n=0,1, \ldots)
$$

whenever $f$ is a function of $\mathrm{cl} V(E)$ with expansion (1.2).

COROLlaRY 1. A sufficient condition that $E_{\Lambda}$ be nonfundamental in $\mathscr{L}^{p}(-\pi \Delta, \pi \Delta)$ $(1 \leqq p<\infty)$ is that $\Delta>\mathscr{D}$.

It is interesting to compare the above corollary with the following theorem of Redheffer [13].

THEOREM A. Let $\phi$ be a function which is positive and continuous on $(1, \infty)$ and which satisfies

(1) $\phi(x) \uparrow \infty$;

(2) $x^{-1} \phi(x) \downarrow 0$;

(3) $\int_{1}^{\infty} x^{-2} \phi(x) d x=\infty$.

Then there is a sequence $\Lambda$ with

$$
\lim _{x \rightarrow \infty} \frac{N[x-\phi(x), x)}{\phi(x)}=0
$$

such that $E_{\Lambda}$ is fundamental in $\mathscr{L}(-\pi, \pi)$.

We suppose that $F$ is an entire function which is not a polynomial and has the gap power series expansion

$$
F(z)=\sum_{n=0}^{\infty} a_{n} z^{\lambda_{n}}
$$


where the sequence $\Lambda=\left\{\lambda_{n}\right\}$ has $\Psi$-density $\mathscr{D}<1$. We define

$$
M(r)=\max |F(z)| \quad(|z|=r)
$$

and, if $I$ denotes a closed interval,

$$
M(r, I)=\max |F(z)| \quad(|z|=r ; \arg z \in I) .
$$

With these definitions we have the following theorem.

THEOREM 2. If $\Delta>\mathscr{D}$, then

$$
\log M(r) \sim \log M(r, I)
$$

outside a set of finite logarithmic measure $\left(^{3}\right)$ provided that $I=I(r)$ varies so that $|I| \geqq 2 \pi \Delta$.

Pólya [12] showed in 1929 that, if $F$ is of finite order and $\Lambda$ has $\mathscr{P}=0$, then

$$
\limsup _{r \rightarrow \infty} \frac{\log M(r, I)}{\log M(r)}=1
$$

provided $|I| \geqq \delta$. Various authors have discussed theorems of this type since that date, in particular Kövari [9] who, writing in 1958, gives references to earlier work. More recently, it is shown in [1] that, if $\Delta>\mathscr{A}$, where

$$
\mathscr{A}=\lim _{q \rightarrow \infty} \limsup _{x \rightarrow \infty} \frac{N[x-q, x)}{q}
$$

and $F$ is a function of finite order, then (3.3) holds with no exceptional set provided $|I| \geqq 2 \pi \Delta$.

4. Some remarks on Theorem 1. (1) The requirement that $\Lambda$ be a sequence of positive integers is not critical in the proof of Theorem 1. If $\Lambda$ is a countable collection of real numbers which can be ordered by increasing magnitude, let $\Lambda^{+}$be the subset of nonnegative elements and $\Lambda^{-}$the subset of negative elements, the latter with their signs reversed. We can then define the $\Psi$-density of $\Lambda$ to be the larger of $\mathscr{D}^{+}$and $\mathscr{D}^{-}$, where these are defined in the obvious way using (2.2).

With this definition, the obvious extension of Theorem 1 holds, provided that

$$
|\lambda-\mu| \geqq \gamma>0,
$$

whenever $\lambda$ and $\mu$ are distinct elements of $\Lambda$. Even this last condition may be relaxed, the critical requirement being merely that a suitable analogue of Lemma 2 (iv) be true.

(2) If it is known that

$$
\Delta>\limsup _{x \rightarrow \infty} \frac{N\left[x-\psi_{0}(x), x\right)}{\psi_{0}(x)},
$$

$\left(^{3}\right)$ I.e. a set $E$ for which $\int_{E} t^{-1} d t<\infty$. 
where $\psi_{0} \in \Psi$ satisfies

$$
\psi_{0}(x) / \log x \rightarrow \infty \quad(x \rightarrow \infty)
$$

then the conclusion of Theorem 1 holds with $\psi(x)=A \psi_{0}(B x)$, where $A$ and $B$ are positive constants.

As a consequence we have, for example, the following result. Let

$$
\mathscr{E}=\lim _{\alpha \rightarrow 1-} \limsup _{x \rightarrow \infty} \frac{N\left[x-x^{\alpha}, x\right)}{x^{\alpha}}
$$

and suppose that $\mathscr{E}<\Delta \leqq 1$. Then, for each $p(1 \leqq p \leqq \infty)$, there are constants $A$ and $B(B<1)$, which depend only on $\Lambda$ and $\Delta$, such that

$$
\left|a_{n}\right| \leqq \exp \left(A \lambda_{n}^{B}\right)\|f\|_{p} \quad(n=0,1, \ldots),
$$

whenever $f$ is a function of $\mathrm{cl} V\left(E_{\Lambda}\right)$ with expansion (1.2).

(3) Given any $\Delta(0<\Delta<1)$ and any $\psi \in \Psi$, there exists a sequence $\Lambda$ of positive integers with $\Psi$-density $\mathscr{D} \leqq \Delta$ such that

$$
\sup _{f \in \operatorname{clV}\left(E_{\Lambda}\right)} \frac{\left|a_{n}\right|}{\|f\|_{p}} \geqq \exp \left(\psi\left(\lambda_{n}\right)\right)
$$

for an infinite set of $n$.

Proof. Let $\psi_{0}(x)=C \psi(x)$, where $C$ is a positive constant to be chosen later. Let

$$
\rho=\left|1-e^{i \pi \Delta}\right| \text {. }
$$

Then $0<\rho<2$ and we may choose $\sigma$ so that $\rho<\sigma<2$. Given a sequence $\left\{n_{k}\right\}$ of positive integers, let $w_{k}=\left[\Delta \psi_{0}\left(n_{k}\right)\right], m_{k}=n_{k}-w_{k}$ and $\mathrm{X}_{k}=m_{k}+\left[\frac{1}{2} w_{k}\right]$. We choose $\left\{n_{k}\right\}$ so that

(1) $m_{k}-\psi_{0}\left(m_{k}\right)>n_{k-1} \quad(k=1,2, \ldots)$;

(2) $\sum_{k=1}^{\infty}(\rho / \sigma)^{w_{k}}<(2 \pi \Delta)^{-1 / p}$.

We now obtain $\Lambda$ by deleting the positive integers which lie outside the intervals $\left[m_{k}, n_{k}\right)(k=1,2, \ldots)$. The sequence $\Lambda$ has $\Psi$-density $\mathscr{D} \leqq \Delta$. Let

$$
f(x)=\sum_{k=1}^{\infty}\left(\frac{1-e^{i x}}{\sigma}\right)^{w_{k}} \exp \left(\text { ixm }_{k}\right)
$$

Then $\|f\|_{p}<1$. Also $f \in \mathrm{cl} V\left(E_{\Lambda}\right)$, its expansion being obtained by expanding each term of (4.1) by the binomial theorem. If $\mathrm{X}_{k}=\lambda_{n}$, we have

$$
\left|a_{n}\right| \geqq A_{0}^{w_{k}} \geqq A_{1}^{\psi_{0}\left(n_{k}\right)} \geqq A_{2}^{\psi_{0}\left(x_{k}\right)}=A_{2}^{C \psi\left(\lambda_{n}\right)} .
$$

The constants $A_{0}, A_{1}$ and $A_{2}$ are all greater than 1, since $\sigma<2$. With an appropriate choice of $C$ we obtain the required result.

5. Proof of Theorem 1. We begin by giving a rough indication of the structure of the proof. First note that the insertion of a finite number of terms into the sequence $\Lambda$ does not alter the truth of Theorem 1. We therefore prove the theorem, 
in the first instance, with $\Lambda$ replaced by $\Lambda_{N}=\left\{\lambda_{N}, \lambda_{N+1}, \ldots\right\}$. Here $N$ is a large positive integer determined by technical considerations.

Given the number $\Delta^{\prime}$ with $\mathscr{D}<\Delta^{\prime}<1$, our aim is to find a $\psi$ in the set $\Psi$ with which we can associate functions $v_{n}(n=N, N+1, \ldots)$ which are uniformly bounded and whose support lies in $\left[-\pi \Delta^{\prime}, \pi \Delta^{\prime}\right]$. Further, the Fourier transforms $V_{n}$ of these functions must satisfy a number of special properties which we discuss below.

Suppose now that $T$ is a trigonometric polynomial with exponents drawn from the sequence $\Lambda_{N}$, say $T(x)=\sum a_{k} \exp \left(i \lambda_{k} x\right)$. Then

$$
\int_{-\pi \Delta^{\prime}}^{\pi \Delta^{\prime}} T(x) v_{n}(x) d x=\sum a_{k} V_{n}\left(\lambda_{k}\right)
$$

In order to obtain an inequality for the coefficient $a_{n}$ from this expression, it is desirable that the term $a_{n} V_{n}\left(\lambda_{n}\right)$ should dominate the sum on the right-hand side whenever $\left|a_{n}\right|$ is not too small compared with the other coefficients. We ensure this by contriving that the functions $V_{n}$ we construct vanish at each point $\lambda_{k}$ (other than $\lambda_{n}$ ) which lies in the interval $\left[\lambda_{N}, 2 \lambda_{n}\right.$ ) and are quite small compared with $V_{n}\left(\lambda_{n}\right)$ in the interval $\left[2 \lambda_{n}, \infty\right)$. The functions $V_{n}$ are related to the function $\psi$ by the estimate $\left|V_{n}\left(\lambda_{n}\right)\right| \geqq \exp \left(-\psi\left(\lambda_{n}\right)\right)$. This explains roughly how the inequality of Theorem 1 is obtained.

The construction of $V_{n}$ is somewhat complex and is organised in the following way. The set $\Lambda_{N}$ is split up into three parts, $S, T$ and $U$. The set $U$ consists of those $\lambda_{k}$ which satisfy $\lambda_{k} \geqq 2 \lambda_{n}$. The set $S$ is a subset of the interval $\left[\lambda_{N}, 2 \lambda_{n}\right)$. It is itself the union of a large number of subsets of $\Lambda_{N}$ constructed with the help of Lemma 1. Each of these subsets includes $\lambda_{n}$ but they are otherwise disjoint. The points of each particular subset will be far apart, and, on either side of $\lambda_{n}$, each subset will look almost like an arithmetic progression from which certain terms have been omitted. Finally, $T$ will consist of those points of $\Lambda_{N}$ which are too close to $\lambda_{n}$ to be included in the union $S$.

The Fourier transform $V_{n}$ is constructed as the product of three others, $R_{n}, P_{n}$ and $Q_{n}$ associated with the sets $S, T$ and $U$ respectively. (In the notation of the proof, $R_{n}(x)$ is $R\left(x-\lambda_{n}\right), P_{n}(x)$ is $P\left(x-\lambda_{n}\right)$ and $Q_{n}(x)$ is $Q\left(x-\lambda_{n}\right)$.) Lemma 2 is used to construct $P_{n}$ as a Fourier transform (of a function with small support) which vanishes at the points of $T$ but is not too small at $\lambda_{n}$. The function $R_{n}$ is a Fourier transform (of a function with support of length slightly less than $2 \pi \Delta^{\prime}$ ) which is not too small at $\lambda_{n}$ but vanishes at the other points of $S$. Its construction is based on the application of Lemma 4 to each of the component sets which make up $S$. Finally $Q_{n}$ will be a Fourier transform (of a function of small support) provided by Lemma 3. It has the property of being quite small at the points of $U$, but not too small at $\lambda_{n}$.

We now embark on the proof proper.

Lemma 1. Suppose the sequence $\Lambda$ has $\Psi$-density $\mathscr{D}$. Let $\delta>\mathscr{D}$ and $\tau<1$. Then there is an $X>0$ and $a \psi \in \Psi$ such that, whenever $X<a<b$, there is a finite collection 
of disjoint, half-open intervals $\left\{I_{1}, I_{2}, \ldots, I_{K}\right\}$ with the following properties:

$$
\begin{aligned}
& \text { (1) }[a, b)=\bigcup_{j=1}^{K} I_{j} ; \\
& \text { (2) } N\left(I_{j}\right) \leqq \delta \psi(b) \quad(j=1,2, \ldots, K) \\
& \text { (3) } \tau \psi(b) \leqq\left|I_{j}\right| \leqq \psi(b) \quad(j=1,2, \ldots, K-1) .
\end{aligned}
$$

Proof. Since $\Lambda$ has $\Psi$-density $\mathscr{D}$, defined by (2.2), there exists a $\psi_{0} \in \Psi$ and an $X>0$ such that

$$
N\left[x-\psi_{0}(x), x\right) \leqq \delta \psi_{0}(x) \quad(x>X) .
$$

Write $\phi(x)=\phi_{1}(x)=x-\psi_{0}(x)$ and let $\phi_{j}(x)=\phi\left(\phi_{j-1}(x)\right)(j \geqq 2)$. For a given value of $x$, write $x_{0}=x$ and $x_{j}=\phi_{j}(x)(j \geqq 1)$. Then $x_{0}>x_{1}>\cdots$. Let $N$ be the smallest positive integer with $(N-1) / N \geqq \tau$.

We now define

$$
\begin{aligned}
\psi(x) & =x-\phi_{N}(x) \\
& =\psi_{0}\left(x_{0}\right)+\psi_{0}\left(x_{1}\right)+\cdots+\psi_{0}\left(x_{N-1}\right) .
\end{aligned}
$$

We must first show that $\psi \in \Psi$, the set $\Psi$ being defined by (2.1).

Since $\psi_{0}(x) \leqq \psi(x) \leqq N \psi_{0}(x)$, it is clear that $\psi(x) \rightarrow \infty \quad(x \rightarrow \infty), x^{-1} \psi(x) \rightarrow 0$ $(x \rightarrow \infty)$ and

$$
\int_{1}^{\infty} x^{-2} \psi(x) d x<\infty
$$

It remains to prove that $\psi(x) \uparrow$ and $x^{-1} \psi(x) \downarrow$.

Since $x^{-1} \phi(x)=1-x^{-1} \psi_{0}(x)$, we have that $x^{-1} \phi(x) \uparrow$. This implies that $\phi(x) \uparrow$ and therefore that $\phi_{j}(x) \uparrow(j \geqq 1)$. Thus $x \leqq y$ implies $x_{j} \leqq y_{j}(j \geqq 1)$. Hence, if $x \leqq y$,

$$
\begin{aligned}
\psi(x) & =\psi_{0}\left(x_{0}\right)+\cdots+\psi_{0}\left(x_{N-1}\right) \\
& \leqq \psi_{0}\left(y_{0}\right)+\cdots+\psi_{0}\left(y_{N-1}\right) \\
& =\psi(y)
\end{aligned}
$$

and therefore $\psi(x) \uparrow$. Further, since $x^{-1} \phi(x) \uparrow$ and $\phi_{j}(x) \uparrow(j \geqq 1)$, we have that

$$
\phi_{j+1}(x) / \phi_{j}(x)=\phi\left(\phi_{j}(x)\right) / \phi_{j}(x)
$$

is monotone increasing. Writing

$$
\frac{\phi_{N}(x)}{x}=\frac{\phi_{N}(x)}{\phi_{N-1}(x)} \cdot \frac{\phi_{N-1}(x)}{\phi_{N-2}(x)} \cdots \cdots \frac{\phi(x)}{x}
$$

it is clear that $x^{-1} \phi_{N}(x) \uparrow$. But $x^{-1} \psi(x)=1-x^{-1} \phi_{N}(x)$, and so $x^{-1} \psi(x) \downarrow$.

This completes the proof that $\psi \in \Psi$.

Given $a$ and $b(X<a<b)$, we let $I_{1}=[b-\psi(b), b)=\left[b_{N}, b_{0}\right)$. For $j \geqq 2$, we define $I_{j}$ inductively by $I_{j}=\left[b_{p_{j}}, b_{p_{j-1}}\right)$ where $p_{j}$ is the smallest $s$ with the property that

$$
b_{p_{j-1}}-b_{s}>\frac{N-1}{N} \psi(b)
$$


terminating the construction at $j=K-1$ if $b_{p_{K}} \leqq a$ and taking $I_{K}=\left[a, b_{p_{K-1}}\right)$. Thus

$$
\left|I_{j}\right|>\frac{N-1}{N} \psi(b) \geqq \tau \psi(b) \quad(j=1, \ldots, K-1) .
$$

But

$$
\left|I_{j}\right|-\psi_{0}\left(b_{p_{j}}\right)=b_{p_{j-1}}-b_{p_{j}+1} \leqq \frac{N-1}{N} \psi(b) \quad(j=1, \ldots, K-1) .
$$

Since $\psi(x) \uparrow$, we have that $\psi_{0}\left(b_{p_{j}}\right) \leqq N^{-1} \psi(b)$, and so

$$
\left|I_{j}\right| \leqq \frac{N-1}{N} \psi(b)+\frac{1}{N} \psi(b)=\psi(b) \quad j(=1, \ldots, K-1) .
$$

Finally, it follows from (5.2) that

$$
N\left(I_{j}\right) \leqq \delta \psi(b) \quad(j=1, \ldots, K) .
$$

LEMMA 2. Let $\mathrm{N}$ be a set of nonzero integers containing at most $N$ elements and with $\mathrm{N} \subset[-a, a]$, where $2 a \geqq N$. Let $l \leqq \pi N a^{-1}$. Then there is a function $p \in \mathscr{L}(-\infty, \infty)$, which is zero outside $(-l, l)$ and which satisfies

(i) $\sup |p(x)| \leqq l^{-1}$;

(ii) $\int_{-\infty}^{\infty}|p(x)| d x \leqq 1$;

and whose Fourier transform $\left(^{4}\right) P$ satisfies

(iii) $P(x)=0(x \in \mathrm{N})$;

(iv) $|P(0)|^{-1} \leqq(1 / \pi N)(2 \pi e / l)^{n}$.

REMARK. The similarity of the constant in (iv) above to that which appears in "Turan's Lemma" (see [15, p. 30]) is not a coincidence. For small values of the parameter $\delta$ figuring in its statement, Turan's Lemma can be obtained from the above result.

Proof. Let $\left\{\nu_{1}, \nu_{2}, \ldots, \nu_{N}\right\}$ be a collection of nonzero integers in $[-a, a]$ which contains N. Consider the product

$$
\begin{aligned}
\Pi(x) & =\prod_{k=1}^{N} \cos \frac{1}{2} l N^{-1}\left\{x-\nu_{k}+\pi N l^{-1}\right\} \\
& =\sum_{k=-N}^{N} c_{k} \exp \left(i x\left(\frac{1}{2} l N^{-1}\right) k\right),
\end{aligned}
$$

in which expression $\sum_{k=-N}^{N}\left|c_{k}\right| \leqq 1$. Take

$$
P(x)=\left(\frac{\sin \frac{1}{2} l x}{\frac{1}{2} l x}\right) \Pi(x) \quad(P(0)=\Pi(0)) .
$$

This is the Fourier transform of the function

$$
p(t)=\sum_{k=-N}^{N} c_{k} \delta_{l}\left(t-\frac{1}{2} l k N^{-1}\right)
$$

(4) I.e. $P(u)=\int_{-\infty}^{\infty} e^{i u x} p(x) d x$. 
where

$$
\begin{aligned}
\delta_{l}(t) & =l^{-1}, & & |t|<\frac{1}{2} l, \\
& =0, & & |t| \geqq \frac{1}{2} l .
\end{aligned}
$$

We have that $p$ is zero outside $(-l, l)$ and

(i) $\sup |p(x)| \leqq l^{-1} \sum_{k=-N}^{N}\left|c_{k}\right| \leqq l^{-1}$;

(ii) $\int_{-\infty}^{\infty}|p(x)| d x \leqq \sum_{k=-N}^{N}\left|c_{k}\right| \leqq 1$.

Further,

(iii) $P\left(\nu_{k}\right)=\left(\sin \frac{1}{2} l v_{k} / \frac{1}{2} l v_{k}\right) \Pi\left(\nu_{k}\right)=0(k=1,2, \ldots, N)$.

It remains to prove (iv). We have

$$
\begin{aligned}
|P(0)|^{-1} & =\prod_{k=1}^{N}\left|\cos \frac{1}{2} l N^{-1}\left(\pi N l^{-1}-\nu_{k}\right)\right|^{-1} \\
& =\prod_{k=1}^{N}\left|\sin \frac{1}{2} l N^{-1} \nu_{k}\right|^{-1} .
\end{aligned}
$$

Since $l \leqq \pi N a^{-1}$,

$$
\left|\frac{1}{2} l N^{-1} \nu_{k}\right| \leqq \frac{1}{2} l N^{-1} a \leqq \pi / 2 \quad(k=1,2, \ldots, N) .
$$

Hence, using the inequality

$$
|\sin x| \geqq 2|x| / \pi \quad(|x| \leqq \pi / 2) ;
$$

we obtain

(iv)

$$
\begin{aligned}
|P(0)|^{-1} & \leqq \prod_{k=1}^{N} \frac{\pi}{2} \cdot \frac{2 N}{l} \cdot \frac{1}{\left|\nu_{k}\right|} \\
& =\left(\frac{\pi N}{l}\right)^{N} \prod_{k=1}^{N}\left|\nu_{k}\right|^{-1} \\
& \leqq\left(\frac{\pi N}{l}\right)^{N}\left\{\left[\frac{N}{2}\right] !\right\}^{-2} \\
& \leqq \frac{1}{\pi N}\left(\frac{2 \pi e}{l}\right)^{N} .
\end{aligned}
$$

Lemma 3 (Ingham [6]). Suppose that $l>0$ and $\varepsilon$ denotes a positive, monotone decreasing function with domain $(1, \infty)$. Then a necessary and sufficient condition that there exist a function $q \in \mathscr{L}(-\infty, \infty)$ which is zero outside $(-l, l)$ and which satisfies

(i) $\int_{-\infty}^{\infty}|q(x)| d x \leqq 1$;

and whose Fourier transform $Q$ satisfies

(ii) $Q(x)=O(\exp (-|x| \varepsilon(|x|)))(|x| \rightarrow \infty)$;

(iii) $Q(0)=1$

is that

$$
\int_{1}^{\infty} \frac{\varepsilon(x)}{x} d x<\infty
$$


REMARK. We use only the sufficiency which is easily proved by taking

$$
Q(x)=\prod_{k=1}^{\infty} \frac{\sin \rho_{k} x}{\rho_{k} x}
$$

where the sequence $\left\{\rho_{k}\right\}$ is chosen appropriately.

LEMMA 4 (INGHAM [4]). Let $\mathrm{N}$ be a finite set of integers which includes zero, and has the property that $|m-n| \geqq \sigma>0$ for each distinct $m$ and $n$ in $\mathrm{N}$. Then there is $a$ function $r \in \mathscr{L}(-\infty, \infty)$ which is zero outside $\left(-\pi \sigma^{-1}, \pi \sigma^{-1}\right)$ and which satisfies

(i) $\int_{-\infty}^{\infty}|r(t)| d t \leqq 1$;

and whose Fourier transform $R$ satisfies

(ii) $R(x)=0 \quad(x \in \mathrm{N}$ but $x \neq 0)$;

(iii) $|R(0)|^{-1} \leqq 2$.

REMARK. Construct the functions $k$ and $K$ of Ingham's paper appropriate to the set $\left\{\nu \sigma^{-1} \mid N \in \nu\right\}$, defining $k(t)$ to be zero for $|t|>\pi$. We may then take

$$
R(x)=\frac{1}{2} \frac{K\left(x \sigma^{-1}\right)}{K(0)}
$$

which implies that

$$
r(t)=\frac{1}{2} \frac{\sigma k(\sigma t)}{K(0)} .
$$

LEMma 5 (L. SCHWARTZ [14]). A necessary and sufficient condition that $E_{\Lambda}$ be free in $\mathscr{L}^{p}(-\pi \Delta, \pi \Delta)$, where $1 \leqq p<\infty$ and $\Delta>0$, is the existence of functions $g_{n}(n=0,1, \ldots)$ in $\mathscr{L}^{q}(-\pi \Delta, \pi \Delta)(1 / p+1 / q=1)$ such that

$$
\begin{aligned}
\int_{-\pi \Delta}^{\pi \Delta} \exp \left(i \lambda_{k} x\right) g_{n}(x) d x & =1, & k=n, \\
& =0, & k \neq n,
\end{aligned}
$$

where $\left\|g_{n}\right\|_{q}$ is the smallest $C_{n}$ which satisfies (1.1).

An analogous result holds for $p=\infty$.

RemarK. The sufficiency is obvious. For the necessity, note that the continuous linear functional $L_{n}$ of $\S 1$ may be extended to $\mathscr{L}^{p}(-\pi \Delta, \pi \Delta)$ by the HahnBanach theorem. An application of the Riesz representation theorem then yields the lemma.

LEMMA 6. Let $\Lambda_{N}=\left\{\lambda_{N}, \lambda_{N+1}, \ldots\right\}$ and suppose that the set $E^{\prime}=E_{\Lambda_{N}}$ is free in $\mathscr{L}^{p}\left(-\pi \Delta^{\prime}, \pi \Delta^{\prime}\right)$ for some $p(1 \leqq p \leqq \infty)$ and some $\Delta^{\prime}>0$, and that $\left|a_{n}\right| \leqq C_{n}^{\prime}\|f\|_{p}^{\prime}$, whenever $f \in \mathrm{cl} V\left(E^{\prime}\right)$ has expansion $(1.2)\left({ }^{5}\right)$.

Then, if $\Delta>\Delta^{\prime}$, the set $E=E_{\Lambda}$ is free in $\mathscr{L}^{1}(-\pi \Delta, \pi \Delta)$ and $\left|a_{n}\right| \leqq C_{n}\|f\|_{1}$, whenever $f \in \mathrm{cl} V(E)$ has expansion (1.2). Here $C_{n} \leqq H \lambda_{n}^{N+1} C_{n}^{\prime}(n \geqq N)$, where $H$ is a constant which depends only on $N, \Delta^{\prime}$ and $\Delta$.

${ }^{(5)}$ In this sentence, cl $V\left(E^{\prime}\right)$ denotes the closure of $V\left(E^{\prime}\right)$ in the space $\mathscr{L}^{p}\left(-\pi \Delta^{\prime}, \pi \Delta^{\prime}\right)$ and $\|\cdot\|_{p}^{\prime}$ denotes the norm of this space. 
Proof. We consider only the case $1 \leqq p<\infty$. When $p=\infty$ there is an analogous argument.

The proof consists of constructing functions $g_{n}(n=0,1, \ldots)$ which satisfy Lemma 5 with $p=1$. The existence of functions $h_{n}(n=N, N+1, \ldots)$ in $\mathscr{L}^{q}\left(-\pi \Delta^{\prime}, \pi \Delta^{\prime}\right)$ with

$$
\begin{aligned}
\int_{-\pi \Delta^{\prime}}^{\pi \Delta^{\prime}} \exp \left(i \lambda_{k} x\right) h_{n}(x) d x & =1, \quad k=n, \\
& =0, \quad k \geqq N \text { but } k \neq n,
\end{aligned}
$$

and $\left\|h_{n}\right\|_{q}^{\prime} \leqq C_{n}^{\prime}(n \geqq N)$ is already assured by Lemma 5 .

We now construct a bounded function $j_{n}$ which vanishes outside $(-l, l)$, where $l \leqq \frac{1}{2} \pi\left(\Delta-\Delta^{\prime}\right)$ and satisfies

$$
\begin{array}{rlrl}
\int_{-l}^{l} \exp \left(i \lambda_{k} x\right) j_{n}(x) d x & =1, & & k=n, \\
& =0, & 0 \leqq k<N .
\end{array}
$$

To do this, take $\mathrm{N}=\left\{\lambda_{k}-\lambda_{n} \mid 0 \leqq k<N\right\}, a=\lambda_{n}$ and $l=\min \left\{\frac{1}{2} \pi\left(\Delta-\Delta^{\prime}\right), \pi N a^{-1}\right\}$ in Lemma 2. We obtain functions $p$ and $P$ with the properties described in the lemma. Now define

$$
j_{n}(x)=\{P(0)\}^{-1} \exp \left(-i x \lambda_{n}\right) p(x)
$$

Then $j_{n}$ satisfies the condition specified above and also

$$
\sup |j(x)| \leqq \frac{1}{\pi N l}\left(\frac{2 \pi e}{l}\right)^{N}
$$

For $n \geqq N$, we now define $g_{n}=j_{n} * h_{n}$. The functions $g_{n}(n \geqq N)$ now satisfy Lemma 5 with $p=1$, and $\left\|g_{n}\right\|_{\infty} \leqq H \lambda_{n}^{N+1}\left\|h_{n}\right\|_{q}^{\prime}$, where $H$ is a constant which depends only on $N, \Delta^{\prime}$ and $\Delta$.

A similar argument may be used to construct the functions $g_{n}(n<N)$.

Proof of Theorem 1. We begin by selecting functions $\Delta^{\prime}, \Delta^{\prime \prime}, \Delta^{m}$ and $\Delta^{\mathrm{iv}}$ of $\mathscr{D}$ and $\Delta$ in such a way that

$$
\mathscr{D}<\Delta^{\mathrm{iv}}<\Delta^{m}<\Delta^{\prime \prime}<\Delta^{\prime}<\Delta \leqq 1,
$$

and

$$
l=\frac{1}{2} \pi\left(\Delta^{\prime}-\Delta^{\prime \prime}\right)<\pi \Delta^{1 \mathrm{v}} .
$$

Let

$$
\rho=\Delta^{\prime \prime} / \Delta^{\prime \prime}>1
$$

and define $h$ to be the smallest positive integer which satisfies

$$
h /(h-1) \leqq \rho .
$$

Further, let

$$
\tau=\Delta^{\mathrm{iv}} / \Delta^{m}<1
$$


We define

$$
\Lambda_{N}=\left\{\lambda_{N}, \lambda_{N+1}, \ldots\right\}
$$

Here $N$ is a constant, depending only on $\Lambda$ and $\Delta$, on which we shall make a number of requirements during the course of the proof.

The body of the proof consists of showing that the set

$$
E_{\Lambda_{N}}=\left\{\exp (i \lambda x) \mid \lambda \in \Lambda_{N}\right\}
$$

is free in $\mathscr{L}^{p}\left(-\pi \Delta^{\prime}, \pi \Delta^{\prime}\right)$.

In Lemma 1, we take $\delta=\Delta^{\mathrm{iv}}$ and $\tau$ as in (5.7). Then there is a $\psi_{0} \in \Psi$ and an $X>0$ for which the conclusions of the lemma hold. Our first requirement on the constant $N$ in (5.8) is therefore that $\lambda_{N}>X$.

Let $\psi_{1}$ be a second function in $\Psi$ which has the property that $\psi_{1}(x) \geqq \psi_{0}(x)$ for each $x>0$ and

$$
\psi_{1}(x) / \log x \rightarrow \infty \quad(x \rightarrow \infty) .
$$

We now define the function $\varepsilon$ by

$$
\varepsilon(x)=A \psi_{1}(4 x) / x
$$

where the constant $A$, which will be specified later, is to depend only on $\Lambda$ and $\Delta$. In view of conditions $(2.1), \varepsilon$ is positive, monotone decreasing and

$$
\int_{1}^{\infty} \frac{\varepsilon(x)}{x} d x<\infty
$$

Further $x \varepsilon(x) \uparrow$. Finally, we define

$$
\psi(x)=B \psi_{1}(2 x)
$$

where, again, $B$ is a positive constant to be specified later and depends only on $\Lambda$ and $\Delta$. Clearly $\psi \in \Psi$.

We now fix a positive integer $k \geqq N$ and write

$$
q=q_{k}=\psi_{0}\left(2 \lambda_{k}\right) ; \mathrm{X}=\mathrm{X}_{k}=\left[q \Delta^{\mathrm{iv}}\right] .
$$

(Since $\psi_{0}(x) \rightarrow \infty(x \rightarrow \infty)$, we make a further minor requirement on $N$ in (5.8) that $\mathrm{X}_{k} \geqq 1(k \geqq N)$.)

From Lemma 1, we obtain that there exists a finite collection of disjoint halfopen intervals $\left\{I_{1}, I_{2}, \ldots, I_{K}\right\}$ such that

$$
\begin{aligned}
\text { (i) } & {\left[\lambda_{N}, 2 \lambda_{k}\right)=\bigcup_{j=1}^{K} I_{j} } \\
\text { (ii) } & N\left(I_{j}\right) \leqq \mathrm{X} \quad(j=1, \ldots, K) \\
\text { (iii) } & \tau q<\left|I_{j}\right| \leqq q \quad(j=1, \ldots, K-1) .
\end{aligned}
$$

Let $I_{s}$ be the interval from this collection which contains $\lambda_{k}$. 
Recalling that $h$ is defined by (5.6), we suppose that $\alpha$ is an integer satisfying $1 \leqq \alpha \leqq h \mathrm{X}$. Then $\alpha$ can be written in the form $\alpha=\beta \mathrm{X}+\gamma$, where $\beta$ and $\gamma$ are integers and $1 \leqq \gamma \leqq \mathrm{X}$. Let $S_{\alpha}$ be that subset of $\Lambda_{N} \cap\left[\lambda_{N}, 2 \lambda_{k}\right)$ which contains precisely the following elements:

(1) $\lambda_{k}$;

(2) the $\gamma$ th smallest element of $\Lambda_{N}$, where this exists, contained in each interval $I_{s+r h+\beta}(r=1,2, \ldots)$;

(3) the $\gamma$ th largest element of $\Lambda_{N}$, where this exists, contained in each interval $I_{s-r h-\beta}(r=1,2, \ldots)$.

In view of (5.13) (iii), the sets $S_{\alpha}(\alpha=1,2, \ldots, h \mathrm{X})$ have the property that

$$
|m-n| \geqq(h-1) \tau q
$$

whenever $m$ and $n$ are distinct elements of $S_{\alpha}$. (The construction of the sets $S_{\alpha}$ is similar to a construction used in [1], where a diagram is available.)

Let $T$ be the set of those elements of $\Lambda_{N} \cap\left[\lambda_{N}, 2 \lambda_{k}\right.$ ) which do not belong to $S=\bigcup_{\alpha=1}^{x h} S_{\alpha}$. Then

$$
T \subset\left[\lambda_{k}-h q, \lambda_{k}+h q\right]
$$

and contains at most $2 \mathrm{X} h$ terms.

Finally, let $U=\Lambda_{N} \cap\left[2 \lambda_{k}, \infty\right)$. Then $\Lambda_{N}=S \cup T \cup U$.

In Lemma 4 we take, for a given value of $\alpha(1 \leqq \alpha \leqq h \mathrm{X}), \mathrm{N}=\left\{\lambda-\lambda_{k} \mid \lambda \in S_{\alpha}\right\}$ and $\sigma=(h-1) \tau q$. In view of (5.14), it follows from the lemma that there exists a function $r_{\alpha}$, zero outside $\left(-\pi \sigma^{-1}, \pi \sigma^{-1}\right)$ with

(i) $\int_{-\infty}^{\infty}\left|r_{\alpha}(t)\right| d t \leqq 1$; and whose Fourier transform $R_{\alpha}$ satisfies

(ii) $R_{\alpha}\left(\lambda-\lambda_{k}\right)=0\left(\lambda \in S_{\alpha}\right.$ but $\left.\lambda \neq \lambda_{k}\right)$;

(iii) $\left|R_{\alpha}(0)\right|^{-1} \leqq 2$.

If we now define $r=r_{1} * r_{2} * \cdots * r_{\mathrm{X} h}$, we have that $r(t)$ is zero for

$$
|t|>\pi \mathrm{X} h / \sigma=\pi h \mathrm{X} /(h-1) \tau q .
$$

But $\mathrm{X}=\left[q \Delta^{\mathrm{iv}}\right]$, from (5.12). Hence $\mathrm{X} \leqq q \Delta^{\mathrm{iv}}$. Therefore, by (5.5)-(5.7),

$$
\frac{\pi \mathrm{X} h}{\sigma} \leqq \frac{\pi h}{h-1} \cdot \frac{\Delta^{\mathrm{iv}}}{\tau}=\frac{\pi h}{(h-1)} \Delta^{m} \leqq \pi \rho \Delta^{m}=\pi \Delta^{\prime \prime} .
$$

It follows that the function $r$ vanishes outside $\left(-\pi \Delta^{\prime \prime}, \pi \Delta^{\prime \prime}\right)$. Further

(i) $\int_{-\infty}^{\infty}|r(t)| d t \leqq 1$.

The Fourier transform of $r$ is $R=R_{1}, R_{2}, \ldots, R_{\mathrm{X} h}$, which satisfies

(ii) $R\left(\lambda-\lambda_{k}\right)=0\left(\lambda \in S\right.$ but $\left.\lambda \neq \lambda_{k}\right)$;

(iii) $|R(0)|^{-1} \leqq 2^{\mathrm{X} h}$;

and, in view of (i),

(iv) $|R(x)| \leqq 1$. 
In Lemma 2 we take $N=\left\{\lambda-\lambda_{k} \mid \lambda \in T\right\}$, with $N=2 \mathrm{X} h, a=h q$ and $l$ as in (5.4). Then $N \leqq 2 a$, and since $\mathrm{X}>q \Delta^{\mathrm{iv}}-1$,

$$
l=\frac{1}{2} \pi\left(\Delta^{\prime}-\Delta^{\prime \prime}\right)<\pi \Delta^{\mathrm{iv}}<\left(\frac{\mathrm{X}+1}{q}\right) \pi \leqq \frac{2 \mathrm{X} \pi}{q}=\frac{N h^{-1} \pi}{a h^{-1}}=\pi N a^{-1} .
$$

The conditions of Lemma 2 are therefore satisfied and so there exists a function $p$, zero outside $(-l, l)$ with

(i) $\int_{-\infty}^{\infty}|p(t)| d t \leqq 1$;

(ii) $\sup |p(t)| \leqq l^{-1}$;

and whose Fourier transform $P$ satisfies

(iii) $P\left(\lambda-\lambda_{k}\right)=0(\lambda \in T)$;

(iv) $|P(0)|^{-1} \leqq C^{2 h \mathrm{x}}$

(where $C=2 \pi e l^{-1}$ ). In view of (i), we also have

(v) $|P(x)| \leqq 1$.

In Lemma 3, we take $l$ as in (5.4) and $\varepsilon$ as in (5.10). There then exists a function $q$, zero outside $(-l, l)$ with

(i) $\int_{-\infty}^{\infty}|q(t)| d t \leqq 1$;

and whose Fourier transform $Q$ satisfies

(ii) $|Q(x)| \leqq E \exp \{-|x| \varepsilon(|x|)\}(|x|>Y)$,

where $E$ and $Y$ are constants which depend only on $l$ and the function $\varepsilon$;

(iii) $Q(0)=1$.

We now take $u=p * q * r$. Then $u$ has Fourier transform $U=P \cdot Q \cdot R$. Finally, we define $v_{k}(x)=\exp \left(i \lambda_{k} x\right) u(x)$ which has Fourier transform $V_{k}(x)=U\left(x-\lambda_{k}\right)$. The function $v_{k}(x)$ is zero for $|x|>\pi \Delta^{\prime \prime}+2 l=\pi \Delta^{\prime}$. Further,

(i) $\sup \left|v_{k}(t)\right| \leqq l^{-1}$;

(ii) $V_{k}\left(\lambda_{j}\right)=0 \quad\left(\lambda_{N} \leqq \lambda_{j}<2 \lambda_{k}\right.$ but $\left.j \neq k\right)$;

(iii) $\left|V_{k}(x)\right| \leqq E \exp \left\{-\left(x-\lambda_{k}\right) \varepsilon\left(x-\lambda_{k}\right)\right\} \quad\left(x>Y+\lambda_{k}\right)$;

(iv) $\left|V_{k}\left(\lambda_{k}\right)\right|^{-1} \leqq F^{\mathbf{x}_{k}} \quad\left(\right.$ where $\left.F=\left(4 \pi e l^{-1}\right)^{2 n}\right)$.

At this stage, we require further of $N$ in (5.8) that

$$
\lambda_{N}>Y
$$

So far we have constructed, for each fixed $k \geqq N$, functions $v_{k}$ and $V_{k}$ with the above properties. In the remainder of the proof, $k$ is no longer fixed.

Consider now an arbitrary trigonometric polynomial with exponents drawn from $\Lambda_{N}$, say $T(x)=\sum_{k=N}^{M} a_{k} \exp \left(i \lambda_{k} x\right)$. We fix our attention on a value of $n$ with the property that

$$
\left|a_{n} V_{n}\left(\lambda_{n}\right)\right|=\max _{N \leqq k \leqq M}\left|a_{k} V_{k}\left(\lambda_{k}\right)\right| .
$$


Then

$$
\begin{aligned}
\int_{-\pi \Delta^{\prime}}^{\pi \Delta^{\prime}}\left|T(x) v_{n}(x)\right| d x & \geqq\left|\int_{-\pi \Delta^{\prime}}^{\pi \Delta^{\prime}} T(x) v_{n}(x) d x\right| \\
& =\left|\sum_{k=N}^{M} a_{k} \int_{-\pi \Delta^{\prime}}^{\pi \Delta^{\prime}} e^{i \lambda_{k} x} v_{n}(x) d x\right| \\
& =\left|\sum_{k=N}^{M} a_{k} V_{n}\left(\lambda_{k}\right)\right| \\
& =\left|a_{n} V_{n}\left(\lambda_{n}\right)+\sum_{2 \lambda_{n} \leqq \lambda_{k} \leqq \lambda_{M}} a_{k} V_{n}\left(\dot{\lambda}_{k}\right)\right| \\
& \geqq\left|a_{n} V_{n}\left(\lambda_{n}\right)\right|\left\{1-\sum_{\lambda_{k} \geqq 2 \lambda_{n}}\left|\frac{V_{n}\left(\lambda_{k}\right)}{V_{k}\left(\lambda_{k}\right)}\right|\right\}
\end{aligned}
$$

Now

$$
\left|V_{n}\left(\lambda_{k}\right)\right| \leqq E \exp \left\{-\left(\lambda_{k}-\lambda_{n}\right) \varepsilon\left(\lambda_{k}-\lambda_{n}\right)\right\} \quad\left(\lambda_{k}>Y+\lambda_{n}\right) .
$$

But, if $\lambda_{k} \geqq 2 \lambda_{n}$, then $\lambda_{k}-\lambda_{n} \geqq \frac{1}{2} \lambda_{k} \geqq \lambda_{n} \geqq \lambda_{N}>Y$ by (5.18). At the time $\varepsilon$ was chosen (i.e. after (5.10)), it was noted that $x \varepsilon(' x) \uparrow$. Hence

$$
\left|V_{n}\left(\lambda_{k}\right)\right| \leqq E \exp \left\{-\frac{1}{2} \lambda_{k} \varepsilon\left(\frac{1}{2} \lambda_{k}\right)\right\} \quad\left(\lambda_{k} \geqq 2 \lambda_{n}\right) .
$$

Moreover, from (5.12) and (5.17),

$$
\left|V_{k}\left(\lambda_{k}\right)\right|^{-1} \leqq F^{\mathrm{x}_{k}} \leqq \exp \left(C \psi_{1}\left(2 \lambda_{k}\right)\right),
$$

since $\psi_{0}(x) \leqq \psi_{1}(x)$. Here $C$ denotes a positive constant which depends only on $\Lambda$ and $\Delta$. Combining (5.21) and (5.22), we have

$$
\begin{aligned}
\left|V_{n}\left(\lambda_{k}\right) / V_{k}\left(\lambda_{k}\right)\right| & \leqq \exp \left\{C \psi_{1}\left(2 \lambda_{k}\right)-\frac{1}{2} \lambda_{k} \varepsilon\left(\frac{1}{2} \lambda_{k}\right)\right\} \\
& =\exp \left\{C \psi_{1}\left(2 \lambda_{k}\right)-A \psi_{1}\left(2 \lambda_{k}\right)\right\}
\end{aligned}
$$

where we have substituted for $\varepsilon$, using (5.10).

By (5.9) we have that

$$
\int_{1}^{\infty} \exp \left(-C \psi_{1}(x)\right) d x<\infty
$$

Hence, if we choose $A=2 C$ in (5.10), we obtain

$$
\begin{aligned}
\sum_{\lambda_{k} \geqq 2 \lambda_{n}}\left|\frac{V_{n}\left(\lambda_{k}\right)}{V_{k}\left(\lambda_{k}\right)}\right| & \leqq \sum_{\lambda_{k} \geqq 2 \lambda_{n}} \exp \left(-C \psi_{1}\left(2 \lambda_{k}\right)\right) \\
& \leqq \int_{2 \lambda_{n}}^{\infty} \exp \left(-C \psi_{1}(x) d x\right) \\
& <1 / 2
\end{aligned}
$$

for $\lambda_{n}>Z$, where $Z$ is a constant which depends only on $\Lambda$ and $\Delta$. The final 
requirement on the constant $N$ of (5.8) is therefore that $\lambda_{N}>Z$. Returning to (5.20), we deduce from (5.23) that

$$
\begin{aligned}
\left|a_{n} V_{n}\left(\lambda_{n}\right)\right| & <2 \int_{-\pi \Delta^{\prime}}^{\pi \Delta^{\prime}}\left|T(x) v_{n}(x)\right| d x \\
& \leqq 2 l^{-1} \int_{-\pi \Delta^{\prime}}^{\pi \Delta^{\prime}}|T(x)| d x,
\end{aligned}
$$

by (5.16). But, by (5.19),

$$
\left|a_{k} V_{k}\left(\lambda_{k}\right)\right| \leqq\left|a_{n} V_{n}\left(\lambda_{n}\right)\right| \leqq 2 l^{-1} \int_{-\pi \Delta^{\prime}}^{\pi \Delta^{\prime}}|T(x)| d x \quad(k=N, \ldots, M),
$$

and so, by (5.22),

$$
\left|a_{k}\right| \leqq 2 l^{-1} \exp \left(C \psi_{1}\left(2 \lambda_{k}\right)\right) \int_{-\pi \Delta^{\prime}}^{\pi \Delta^{\prime}}|T(x)| d x \quad(k=N, \ldots, M) .
$$

The extension of this inequality to the case when $T$ is no longer an element of $V\left(E_{\Lambda_{N}}\right)$ but of $\operatorname{cl} V\left(E_{\Lambda_{N}}\right)$ is trivial.

It now follows from Lemma 6 that

$$
\left|a_{k}\right| \leqq R \lambda_{k}^{N+1} \exp \left(C \psi_{1}\left(2 \lambda_{k}\right)\right)\|f\|_{1} \quad(k=0,1, \ldots),
$$

whenever $f \in \operatorname{cl} V\left(E_{\Lambda}\right)$ has expansion (1.2). Here $R, N$ and $C$ are constants which depend only on $\Lambda$ and $\Delta$. In view of (5.9), there is a constant $S$, which again depends only on $\Lambda$ and $\Delta$, such that

$$
R \lambda_{k}^{N+1} \leqq \exp \left(S \psi_{1}\left(2 \lambda_{k}\right)\right) \quad(k=0,1, \ldots) .
$$

If we define the constant $B$ of (5.11) by $B=S+C$ we then obtain that

$$
\left|a_{k}\right| \leqq \exp \left(\psi\left(\lambda_{k}\right)\right)\|f\|_{1} \quad(k=0,1, \ldots) .
$$

Theorem 1 is therefore proved for the case $p=1$. Since the general case follows from Hölder's inequality, the proof is thus complete.

6. Proof of Theorem 2. In addition to the notation concerning entire functions which was introduced in $\S 3$, we also require the following. Let $\mu(r)$ denote the modulus of the maximum term of (3.2) and let $v(r)$, the "central index", denote the largest value of $\lambda_{n}$ for which this maximum is attained. Clearly $\mu(r) \leqq M(r)$, but also

LEMMA 7 (VALIRON [16]). Outside a set of finite logarithmic measure

$$
M(r) \leqq \mu(r) \log \mu(r) .
$$

LEMMA 8 (KöVARI [10]). Let $\phi$ be any positive monotone increasing function defined on $(0, \infty)$ which has

$$
\int_{0}^{\infty} \frac{d x}{\phi(x)}<\infty
$$

Then, outside a set of finite logarithmic measure,

$$
\nu(r) / \phi(\log v(r))<\log \mu(r) .
$$


It suffices to prove Theorem 2 when $I=[\gamma-\pi \Delta, \gamma+\pi \Delta]$ and $\gamma=\gamma(r)$ varies in an arbitrary fashion.

For a given value of $r$, we apply Theorem 1 with $p=\infty$ to

$$
F\{r \exp (i(\theta+\gamma))\}=\sum\left(a_{n} r^{\lambda_{n}} \exp \left(i \gamma \lambda_{n}\right)\right) \exp \left(i \theta \lambda_{n}\right),
$$

which is considered as a function of $\theta$. We obtain that there exists a $\psi \in \Psi$ such that

$$
\left|a_{n} r^{\lambda_{n}}\right| \leqq \exp \left(\psi\left(\lambda_{n}\right)\right) \max _{\theta \in I}\left|F\left(r e^{i \theta}\right)\right| \quad(n=0,1, \ldots) .
$$

In particular, $\mu(r) \leqq \exp \{\psi(\nu(r))\} M(r, I)$. Hence

$$
\log \mu(r) \leqq \psi(\nu(r))+\log M(r, I) .
$$

Let $\psi_{1}$ be a second function in $\Psi$ with the property that $\psi(x)=o\left(\psi_{1}(x)\right)$. Then

$$
\log \mu(r) \leqq o\left\{\psi_{1}(\nu(r))\right\}+\log M(r, I) .
$$

Choose $\phi$ so that $\phi(\log x)=x / \psi_{1}(x)$. Then $\phi$ is positive, monotone increasing and satisfies (6.1) because

$$
\int_{0}^{\infty} \frac{d y}{\phi(y)}=\int_{1}^{\infty} \frac{d x}{x \phi(\log x)}=\int_{1}^{\infty} \frac{\psi_{1}(x) d x}{x^{2}}<\infty,
$$

by (2.1). We therefore obtain from Lemma 8 that

$$
\psi_{1}(\nu(r))=\nu(r) / \phi(\log \nu(r))<\log \mu(r),
$$

outside a set of finite logarithmic measure. Using this result in (6.2) we have that, outside a set of finite logarithmic measure,

$$
(1+o(1)) \log \mu(r) \leqq \log M(r, I) .
$$

But, by Lemma 7,

$$
\log \mu(r) \sim \log M(r),
$$

outside a set of finite logarithmic measure. Together, (6.3) and (6.4) yield that

$$
\log M(r) \sim \log M(r, I),
$$

outside a set of finite logarithmic measure, as required.

\section{REFERENCES}

1. J. M. Anderson and K. G. Binmore, Coefficient estimates for lacunary power series and Dirichlet series. II, Proc. London Math. Soc. (3) 18 (1968), 49-68. MR 36 \#6624.

2. A. Beurling and $P$. Malliavin, On the closure of characters and the zeros of entire functions, Acta Math. 118 (1967), 79-93, MR 35 \#654.

3. K. G. Binmore A trigonometric inequality, J. London Math. Soc. 41 (1966), 693-696. MR 34 \#559.

4. A. E. Ingham, $A$ further note on trigonometrical inequalities, Proc. Cambridge Philos. Soc. 46 (1950), 535-537. MR 12, 255; MR 12, 1002. 
5. A. E. Ingham, Some trigonometrical inequalities with applications to the theory of series, Math. Z. 41 (1936), 367-379.

6. - A note on Fourier transforms, J. London Math. Soc. 9 (1934), 29-32.

7. J.-P. Kahane, Sur les fonctions moyenne-périodiques bornées, Ann. Inst. Fourier Grenoble 7 (1957), 293-314. MR 21 \#1489.

8. ——, "Travaux de Beurling et Malliavin," in Séminaire Bourbaki, 1961/1962, Exposé 225, Benjamin, New York, 1966, MR 33 \#5420i.

9. T. Kövari, On theorems of G. Pólya and P. Turán, J. Analyse Math. 6 (1958), 323-332. MR 21 \#723.

10. - On the Borel exceptional values of lacunary integral functions, J. Analyse Math. 9 (1961/62), 71-109. MR 25 \#3174.

11. N. Levinson, Gap and density theorems, Amer. Math. Soc. Colloq. Publ., vol. 26, Amer. Math. Soc., Providence, R. I., 1940. MR 2, 180.

12. G. Pólya, Untersuchungen über Lücken und Singularitäten von Potenzreihen, Math. Z. 29 (1929), 449-640.

13. R. Redheffer, Elementary remarks on completeness, Duke Math. J. 35 (1968), 103-116. MR 37 \#687.

14. L. Schwartz, Approximation d'une fonction quelconque par des sommes d'exponentielles imaginaires, Ann. Fac. Sci. Univ. Toulouse (4) 6 (1943), 111-176. MR 7, 437.

15. P. Turan, Eine neue Methode in der Analysis und deren Anwendungen, Akadémiai Kiadó, Budapest, 1953. MR 15, 688.

16. G. Valiron, Lectures on the general theory of integral function, Toulouse, 1923.

State University of New York,

Albany, New York 12203

LONDON SCHOOL OF ECONOMICS,

LONDON, ENGLAND 